\title{
Printer Device
}

National Cancer Institute

\section{Source}

National Cancer Institute. Printer Device. NCI Thesaurus. Code C50120.

An electronic device that is designed to transfer text or images to paper or other substrate. 